\title{
THE TECHNOLOGY MATRIX - A TOOL-BASED METHOD FOR TECHNOLOGY SCOUTING
}

\author{
Marco Peter, Stella Gatziu Grivas, Daniel Horn, Florian Rüegg, Renato Barba and Manuela Graf \\ School of Business, University of Applied Sciences and Arts Northwestern Switzerland \\ Riggenbachstrasse 16, 4600 Olten, Switzerland
}

\begin{abstract}
The digital transformation of businesses is crucial when initiating business model innovations and using technology as added value. The difficulty today for businesses is to keep track of emerging technology trends that are relevant and could significantly support their digitalization process. Especially for small and medium-sized enterprises, which often do not have the resources for a proper investigation of emerging technologies. This research paper presents the technology matrix, a new tool-based method of scouting technology trends, based on the relevant factors business impact, project size, and feasibility of the incorporation of the technologies. Existing technology scouting tools on the markets where analyzed on their strength and weaknesses and best practices were incorporated into the developed technology matrix. Furthermore, expert interviews have led to important inputs when designing the technology matrix.
\end{abstract}

\section{KEYWORDS}

Technology Trends, Technology Scouting, Digital Transformation

\section{INTRODUCTION}

According to the latest data analysis from the federal office for statistics in Switzerland, almost $99.7 \%$ of all enterprises in Switzerland are small and medium-sized enterprises (SMEs) (Bundesamt für Statistik BFS 2017). Therefore, SMEs have a huge impact on the Swiss economy, consequently also in terms of digital transformation. To digitally transform an established business, the companies often have to change their current organization fundamentally. Needed resources, a good holistic understanding, and a clear strategy are necessary to be successful (Kreutzer et al. 2018). Moreover, digital transformation means changing existing business models and most of the time also enable new ones by applying digital technologies.

Quite often SMEs are struggling with the digital transformation. As a study of 1'854 Swiss-based companies has shown, many companies are not satisfied with the outcome of initiatives in the field of digital transformation (Peter 2017). Especially since there are limited resources. SMEs usually cannot invest enough time to tackle the problems of a transformation on their own. Despite the fact that there is a lack of resources, according to the study, they can overcome these obstacles by applying frameworks, which are tailored to their specific needs. As well as working together with external partners to kick-start a transformation project.

There are a lot of frameworks already established on the market but most of them are not suitable for SMEs (Peter et al. 2018). An aspect to be able to foster SMEs with useful tools for digital transformation is to provide a method of how they can analyze the wide range of new technologies for their individual use cases. Even though there are many well-known methods, such as the SWOT-Analysis (Pelz 2004), the PESTEL-Analysis (Theobald 2016), the Porter's Five Forces-Analysis (Chan Kim \& Mauborgne 2005), or the Force Fields Analysis (Baulcomb 2003), which tackle the topic of external analysis, many of them lack to consider the impact of new technologies on a company or industry. A well-defined framework or tool that uses visual representation to support SMEs when defining their digital transformation strategy would be an efficient method. Nevertheless, such a method has not yet been published.

This research project followed the Design Science approach by Hevner et al. (2004). Accordingly, the five phases of problem awareness, suggestion, development, evaluation, and conclusion were carried out. Regarding the awareness phase, the goal of the work was properly outlined by defining research questions and a literature review was carried out in order to investigate the knowledge base. Besides the literature 
review, four expert interviews regarding technology management were conducted. This helped to cover the questions regarding the behavior of Swiss SME. After collecting sufficient information on existing technology radars or similar methods as wells as gaining insight on the preferences and behavior of Swiss SMEs, a newly adapted technology matrix was designed. Nevertheless, an objective of this research project from the very beginning has been that this newly developed tool is usable not only for Swiss SMEs but for any SMEs. The phases suggestion, development, and evaluation were done in an iterative manner, in order to bring the insights from the evaluations into the final tool. The evaluations were carried out in collaboration with experts, where the artifact was presented and discussed in a semi-structured interview form.

\section{OVERVIEW AND COMPARISON OF EXISTING TECHNOLOGY MATRIX'S}

There are different approaches to scouting new technologies, which could help SMEs to find relevant tools to support their digital transformation. To get the key parts and takeaways over the existing frameworks, the available and most relevant ones on the market were analyzed by three different aspects Intended use, Strength, and Weakness, as shown in Table 2.

Table 1. Comparison of Existing Technology Matrixes

\begin{tabular}{|l|l|l|l|l|}
\hline $\begin{array}{l}\text { Name of the } \\
\text { framework }\end{array}$ & Intended use & Strength & Weakness & $\begin{array}{l}\text { Key parts and } \\
\text { takeaways }\end{array}$ \\
\hline $\begin{array}{l}\text { Technology Portfolio } \\
\text { Analysis } \\
\text { (Gatzweiler 2013) }\end{array}$ & $\begin{array}{l}\text { Strategic Technology } \\
\text { Management }\end{array}$ & $\begin{array}{l}\text { Long-term strategic } \\
\text { view. } \\
\text { Simple Matrix }\end{array}$ & $\begin{array}{l}\text { Complex dimensions. } \\
\text { Hard to define } \\
\text { technology } \\
\text { attractiveness. }\end{array}$ & $\begin{array}{l}\text { Simple output but hard } \\
\text { to get the input }\end{array}$ \\
\hline $\begin{array}{l}\text { The Technology Radar } \\
\text { (Rohrbeck et al. 2006) }\end{array}$ & $\begin{array}{l}\text { Transparent internal } \\
\text { communication and } \\
\text { vision }\end{array}$ & $\begin{array}{l}\text { Is meant to be updated } \\
\text { periodically (agile) }\end{array}$ & $\begin{array}{l}\text { Specific for a } \\
\text { technology-based } \\
\text { company }\end{array}$ & $\begin{array}{l}\text { Good criteria's inside } \\
\text { the paper }\end{array}$ \\
\hline $\begin{array}{l}\text { Technology Radar } \\
\text { (Parsons et al. 2018) }\end{array}$ & $\begin{array}{l}\text { Complete overview } \\
\text { from a Technology } \\
\text { perspective }\end{array}$ & $\begin{array}{l}\text { Big picture of all the } \\
\text { Technologies }\end{array}$ & $\begin{array}{l}\text { Specific for a } \\
\text { technology-based } \\
\text { company }\end{array}$ & $\begin{array}{l}\text { A good approach to } \\
\text { classify the technologid }\end{array}$ \\
\hline
\end{tabular}

The technology portfolio analysis from Gatzweiler (2013) supports strategic technology management. It uses a graphical representation of a two-dimensional matrix in which actual and future situations are displayed. In doing so, external (not influenceable by the company) and internally (influenceable by the company) parameters are combined with a view of future technology development. From the resulting constellations, recommendations for the allocation of R\&D funds as well as the company's technology strategy can be derived. The goal of the technology portfolio analysis is to evaluate new, used, or alternative technologies to support strategic decision-making. This approach passes through four consecutive steps: technology identification, determining the current state (technology attractiveness and resource strength), the temporal transformation of the technology portfolio and the selective strategy recommendations. As part of technology identification, the technologies already used in the company are identified. These form the subject of the valuation or comparison. In a two-dimensional matrix, the technologies used in a company or in a particular product or service are mapped individually. The matrix corresponds to the technology portfolio and uses the dimensions of technology attractiveness and resource strength.

The technology portfolio analysis can help to compare the company's own technologies with known alternatives. From this, it is possible to deduce how attractive it is to pursue the current technology strategy in comparison with investing in another, more attractive future technology. If the matrix of a particular technology is supplemented with the identified complementary or substitution alternatives, this gives an overview of how attractive your own technology portfolio will be in the future in comparison to possible alternatives. This comparative assessment can serve as the basis for further strategic decisions. Finally, recommendations for action on the specific design of $R \& D$ programs are given. For this purpose, the so-called standard strategies or selective strategy recommendations are derived from the portfolio. The standard strategies differentiate between investment and divestment strategies. 
The method from Rohrbeck et al. (2006) uses a similar approach. It goes through different steps: identification of potential technologies, selection, assessment, and dissemination of the generated information to the stakeholder inside the company. Deutsche Telekom AG and Cisco already successfully used this approach. Boe-Lillegraven and Monterde (2015) mention that there are three steps in the scouting process: identification step, selection step, and a verification step. Within the identification step, new technologies are submitted by an international network of technology scouts. Moreover, the company itself can define its scouts, which are collecting inputs together with customers, partners, academia, and other stakeholders. As part of the selection phase, a radar team, as well as a panel of senior technical experts on a quarterly basis, review the submissions. The selection is built upon the concepts of technology, impact, and novelty. In the verification step, the selection undergoes a more detailed analysis led by a technology scout. A short technology profile is written for an executive audience, where the technology is put in a business context. The criteria business and technical rank the relevance of each derived technology for the company. The assessment criteria are based on two factors: market opportunity and implementation risk. Finally, the company's Technology Radar gets published frequently with updated technologies along with their maturity, their place in the value chain and their relevance rated by the assessment.

There are also technology radars from companies, such as the open source technology radar from ThoughtWorks (Parsons et al. 2018). Zalando has already put it into practice and adapted it to stay aware of new technologies and tools, which would support their software development team the most. The Radar consists of quadrants, which divide the so-called blips (a blip is a technology or technique that is changing in the field of software development) into the following four categories: programming languages and frameworks, tools, platforms, and techniques. After the blips are assigned to their corresponding quadrant or topic area, they need to be aligned with the rings, which are: adopt, trial, asses, and hold. The closer a blip is to the center of the radar, the sooner it can be adopted. The blips, which are assigned to the most inner ring (adopt) are technologies, tools, or platforms that already should be adopted within the company by now. The trial ring represents the blips that can be used on a trial basis but are not completely proven as the ones in the adopt ring. The technologies, which should be analyzed more closely are placed within the assess ring. Unless they fulfill the company's criteria, those blips do not reach the trial phase. In the outer area of the radar, the hold ring, new technologies introduced to the industries, which yet are not mature enough to use, are placed. These tools have the potential to become relevant in the future.

All three frameworks define different steps or phases, which describe a technology's level of acceptance within a company. To gather information about new technologies, there still need to be scouts or experts involved, in the process of looking out for new tools. If a „radar team“ exists, the phase of technology identification starts with a definition of technology or business needs within the company.

With that input, a technology exploration can be started, either externally or existing ones within the company could be analyzed, whether they still cover the company's needs. For a tool assessment, a graphical depiction could help to structure the findings. Some existing frameworks rank their findings in either concept of Technology, Impact, and Novelty, or dimensions of technology attractiveness and resource strength. Dimensions for supporting and fulfilling the needs of SMEs will be defined further in the document. In all frameworks, experts need to be called in for assessing the result of the findings. In this phase, the rewards and risks of the potential technology need to be further investigated and how the future technology portfolio will differentiate from the current one.

Finally, strategies for investments and divestments for technologies and tools need to be defined. A final graphic could illustrate the company's current state of digitalization with the derived technologies, which can be placed in different phases that express the level of adoption.

\section{THE TECHNOLOGY MATRIX}

The aim of our research and the focus of this paper is a technology matrix which supports through visualization of the impact of different technologies on a company. The following subsections describe the approach and the research results for the developed technology matrix. 


\subsection{Requirements for the Technology Matrix}

In order to develop the technology matrix, requirements, a technology matrix should incorporate, were defined, as listed in Table 2. Thus, the goal of this artifact takes various factors from the applying SME into consideration and in return deliver a clear output showing the most relevant technologies for the company.

Table 2. Requirements for the Artifact Technology Matrix

\begin{tabular}{|l|l|}
\hline Requirement & Description \\
\hline Usability & $\begin{array}{l}\text { As the company should be able to do the assessment by itself, the process of the tool should be } \\
\text { self-explanatory. Thus, a clear structured and easy-to-use process is an essential requirement for the } \\
\text { technology matrix. In addition, the questions leading through the process should be meaningful for } \\
\text { the applying company and the process should be done within a reasonable period. }\end{array}$ \\
\hline $\begin{array}{l}\text { Validity of the } \\
\text { results }\end{array}$ & $\begin{array}{l}\text { The technologies shown in the technology matrix can be traced back and the reason for their result } \\
\text { can be reenacted. }\end{array}$ \\
\hline Comparability & $\begin{array}{l}\text { Also, the results have to be comparable. Hence, the same input data will always lead to the same } \\
\text { result. This will also make the model behind the tool comparable to similar models in that field. }\end{array}$ \\
\hline Self-developing & $\begin{array}{l}\text { The tool should evolve over time as numerous SMEs are applying it. Hence, factors that are static at } \\
\text { the beginning (e.g. project size) can be adapted in the future, based on learnings gained during the } \\
\text { process. }\end{array}$ \\
\hline Sector-relation & $\begin{array}{l}\text { One of the most important factors that have come up during the literature review was that the tool has } \\
\text { to consider the different needs of sectors. As for example, the ICT sector has other needs for } \\
\text { technologies as the hotel industry. }\end{array}$ \\
\hline Multidimensional & $\begin{array}{l}\text { As several important factors need to be displayed in the visual tool, the selected format should be } \\
\text { able to show at least three dimensions. }\end{array}$ \\
\hline
\end{tabular}

\subsection{Development of the Technology Matrix}

After the conduction of an expert interview, it has been decided that the visualization tool needed to support multiple dimensions, in order to fulfill the need of SMEs in their technology trend exploration. The dimensions used are described as the feasibility of a possible technology project and the business impact caused by such a project. In addition, the project size of a possible implementation project is displayed by the size of the respective 'bubble'. As more than one dimension needs to be represented, a radar was deemed not the most suitable option, which usually is used for technology visualization. Instead, a matrix has been selected. An example of the resulted visualization output from the technology matrix is shown in Figure 1.

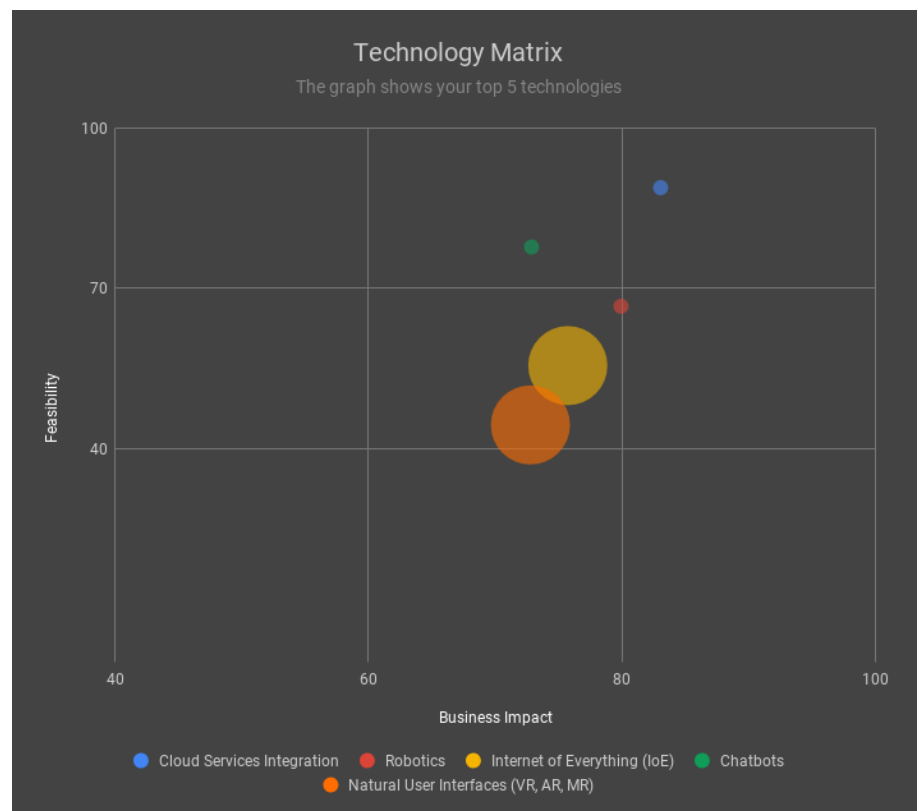

Figure 1. Example of the Technology Matrix, a Technology Trend Visualization 


\subsubsection{Feasibility}

The feasibility is shown on the X-axis and is composed of the factors maturity (of the technology), the complexity (of a possible implementation), and the internal know-how.

A technology's maturity is evaluated with external sources and rated using the numbers 1 (Adolescent), 2 (Early Adoption), or 3 (Mainstream). The ratings are adopted from an article that was published in the year of 2016 by Atos, a big IT service provider, about tech trends with technologies that will impact businesses (Atos Societas Europea 2016). In combination with that, another more extensive report about digital disruption called 'Digital Shockwaves in Business' was published as a part of the Journey 2020 by Atos (2017). Furthermore, the very famous and popular Hype Cycle for Emerging Technologies from Gartner (Panetta 2017) was taken into account by deciding how mature a technology really is. In addition, the article about emerging technology trends from EY (Kimber et al. 2015) was considered as another source.

An external expert, such as a consultant, evaluates the second factor. By completing a predefined form, the expert can indicate if the individual technologies are complex when incorporating within the business. The expert can choose between low, medium, or high related to the complexity.

The last factor, the internal know-how, is assessed by various questions, which have to be answered by the company. For each technology, the company can choose its level of knowledge (e.g. 'training sessions planned', 'technology already in use', 'no knowledge', etc.). Depending on the chosen knowledge level, the internal know-how is rated with either 1,2 , or 3 points.

As all the three factors are rated with numbers from 1 to 3 , the maximum score per technology is 9 . An extract from the tool regarding the feasibility weighting is shown in Figure 2.

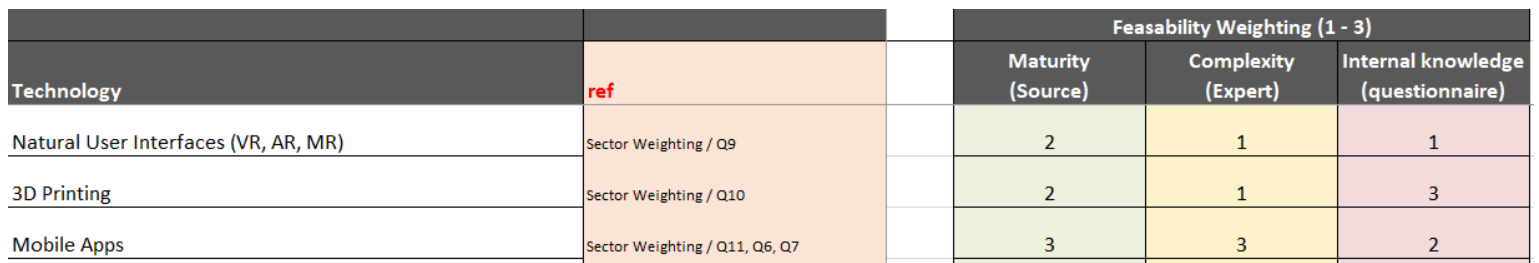

Figure 2. Feasibility weightings

Next, the feasibility needs to be calculated. The formula is as follows:

Key table:

score $i=$ reached score

score $n=$ score $_{\max }$

$$
\frac{100 \times \sum_{\mathrm{n}}^{1}(\text { score } \mathrm{i})}{\text { score } \mathrm{n}}
$$

100, representing the maximum score, is divided by the maximum possible feasibility score (9), and multiplied with the reached score of all factors ([1 to 3]+[1 to 3]+[1 to 3]). For reasons of simplicity, the feasibility is visually split up into three parts within the technology matrix representing a low feasibility, a medium feasibility, and a high feasibility.

\subsubsection{Business Impact}

On the Y-axis of the technology matrix, the business impact is shown. The value for this dimension stems from two factors: the sector the company operates in and the result from the questionnaire, which is filled in by the company. The formula for the calculation of the business impact is as follows:

Key table:

sector score $i=$ reached score, based on sector

sector score $n=$ sector score $_{\max }$

questionnaire score $i=$ reached score, based on questionnaire

questionnaire score $n=$ questionnaire score $_{\max }$

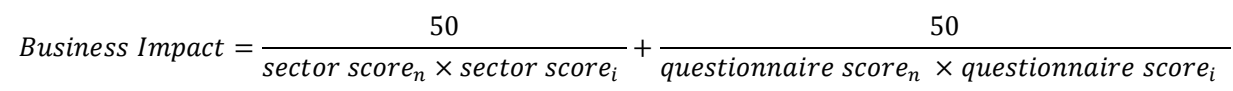


The score for the sector is based on a pre-defined weighting determining the importance of each technology for the respective sector. Initially, this weighting is backed with sources for each sector. In general, all sectors are heading to similar goals, such as automation of processes, using robotics and artificial intelligence to derive more efficient business models and using cloud-based services and analytics to in order to cope with big data. Nevertheless, each sector has special needs regarding technologies. The ICT sector, as the most digitalized sector, strives especially for digital workplaces, development, and usage of more complex technologies such as cognitive computing and the internet of everything (Ewaldsson 2016). Hotels, on the other hand, need to focus on providing better customer services which can be realized through technologies such as chat bots, social media platforms, and automation in form of the internet of things to enable automated check-in and payment processes for instance (Marques 2017). Technologies such as autonomous vehicles could significantly change the industry of manufacturers and retailers into more efficient ways to distribute products. Also, more efficient production processes could be shaped thanks to the combination of robotics and artificial intelligence (Paine 2018).

For a productive version of the technology matrix, this weighting could also be defined by the different inter-trade organizations and be either statically or dynamically. The score per technology is defined by the numbers 1 (low) to 5 (high) and adds $50 \%$ to the total value of the business impact, as the literature review showed the difference between the various sectors. The other half of the business impact is determined by a questionnaire, which is filled in by the company.

For each technology, an ideal response (benchmark) is defined, which can be described as a pattern. The respective questionnaire answers are compared to these technology patterns and the deviations from this ideal response are determined. Eventually, the technology with the least deviation ranks first. The means for the different deviations of each technology are calculated with a weighted arithmetic mean. For every response, there can be an absolute deviation value between zero and maximum four. Furthermore, the total of every deviation is multiplied by a different weight. Each answer with a deviation of zero (which means the perfect match) is multiplied by 8 . The ones with deviation 1 by 4 , deviation 2 by 1 and all the answers with a deviation higher than 2 are multiplied by zero, which means they get ignored. The formula for this calculation is as follows:

Key table:

weighted arithmetic mean $\bar{x}=$ mean of all deviations for a technology

weight $w_{n}=$ weight of a deviation

deviation $d_{n}=$ deviation of an answer to the pattern

$$
\bar{x}=\frac{w_{0} * d_{0}+w_{1} * d_{1}+w_{2} * d_{2}+w_{3} * d_{3}+w_{4} * d_{4}}{w_{0}+w_{1}+w_{2}+w_{3}+w_{4}}
$$

If every answer is the perfect match for one technology, there is a possible maximum weighted arithmetic mean. Which then also means the highest business impact. This is calculated as follows:

Key table:

maximum possible weighted arithmetic mean $\max \bar{x}=$ maximum possible mean for a technology

number of questions (answers) with zero deviation $i=$ number to calculate the maximum mean

$$
\max \bar{x}=\frac{i}{w_{0}+w_{1}+w_{2}+w_{3}+w_{4}}
$$

Summarizing, with these two numbers the business impact can be calculated with the following formula:

$$
\text { Business impact }=50 * \frac{\bar{x}}{\max \bar{x}}
$$

\subsubsection{Project Size}

The third dimension is the size (or effort) of a possible implementation project. Values for this dimension can be 1 (small), 2 (medium), or 3 (large). These values are static and are based on assumptions for this paper. Ideally, SMEs who have applied this tool will give feedback on their implemented projects afterward. Thus, in a productive version, the numbers regarding the project size are dynamically adapted based on experience. Thus, the matrix is self-developing as the number of applying users is growing. 


\subsubsection{Interpretation of the Technology Matrix}

The matrix is roughly divided into 9 fields, representing a low, medium, or high business impact of a technology or feasibility in regards to potential implementation projects. Thus, a company applying the matrix should focus on technologies placed in the upper right corner. On the other hand, technologies located on the bottom left have a low impact on the respective business and are difficult to implement. Due to this, it is recommended to avoid these technologies at current.

In addition to the above factors, the size of the bubble has to be considered as it reflects the size of a possible implementation project. For example, an easy to implement technology with medium business impact could cause a quick win and therefore create momentum for a digital transformation initiative within the company. Small bubbles with relatively high impact can be considered as so-called 'low hanging fruits'.

\subsubsection{Walkthrough of the Tool Technology Matrix}

The exploration process starts with a questionnaire, which needs to be filled out by the SME. It assesses the current situation within the company in terms of internal data concerning the company structure, client data, and data related to the offering. The results of the questionnaire will influence the dimension business impact later on. In order to have a sector-based output, the company needs to fill in their company's sector.

After the questionnaire is filled out, the results are listed in the deviation calculation, where the difference between the as-is situation within the company is compared to the optimal situation in order to incorporate the listed technologies per each deviation. In the meantime, the feasibility weightings need to be assessed. Since the maturity of each technology is set statically, only the complexity and internal knowledge need to be filled out for each technology listed. Experts do this part. The data needed to show relevant technologies in the matrix is now complete and the results are shown within the technology matrix.

\section{EVALUATION}

To evaluate the developed technology matrix as part of this research project, seven tests were performed with SMEs acting as pilots. These pilots were guided through the technology scouting process. By observation technique, the results were noted and later on integrated into the technology matrix tool. The observation was used to evaluate the tool on four key performance indicators (KPI): usability, meaningfulness of the questions, the validity of the results, and the elapsed time. The KPI's are listed in Table 3. The described technology matrix in section 3 is the final outcome of this research project.

Table 3. Evaluation Key Performance Indicators

\begin{tabular}{|l|l|l|}
\hline KPI & Description & Measure \\
\hline Usability & The process is self-explanatory and easy to use & Level of support needed \\
\hline Meaningful questions & $\begin{array}{l}\text { Questions asked during the process were suitable in the context } \\
\text { of the desired result }\end{array}$ & Pilot feedback \\
\hline Validity of result & The delivered results make sense and are comprehensive & Pilot feedback \\
\hline Elapsed time & How much time was spent from start to end & elapsed time in minutes \\
\hline
\end{tabular}

\section{CONCLUSION}

The goal of this research paper is to analyze possibilities for scouting and evaluating current technology trends, which could be relevant particularly for SMEs. SMEs would especially profit from such a tool-based method since they usually do not have the financial resources to hire experts such as consultants. Furthermore, current technology scouting tools are providing support rather for major companies than for SMEs that rely on uncomplicated processes, which do not cause high costs and time efforts. After the analysis of existing methods and frameworks, a technology scout has been designed in the form of a matrix, which weights technologies visually according to their business impact and feasibility in terms of business integration and depicts the project size for each technology. The evaluation of the technology matrix showed a successful and simple usage for SMEs as well as an added value compared to current technology scouting 
tools when scouting for new technology trends, which could play a relevant role for businesses. Thus, this research shows clear benefits for any SMEs when using this technology matrix tool: easy to use, transparency of the output and its process to reach those outputs, the tool can be reused in the future by the SME, and sector specifics are taken into consideration. Concluding, this tool can be applied by any SME in order to facilitate or improve its technology scouting capabilities.

\section{REFERENCES}

Atos, 2017. Journey 2020 - Digital Shockwaves in business. ascent. Available at: https://atos.net/content/minisites/journey-2020/index.html [Accessed October 20, 2018].

Atos Societas Europea, 2016. Technologies that will Impact your Business. ascent, p.28. Available at: https://de.scribd.com/document/367974180/ALO2016-Trends [Accessed October 25, 2018].

Baulcomb, J.S., 2003. Management of change through force field analysis. Journal of Nursing Management, 11(4), pp. $275-280$.

Boe-Lillegraven, S. \& Monterde, S., 2015. Exploring the cognitive value of technology foresight: The case of the Cisco Technology Radar. Technological Forecasting and Social Change, 101, pp.62-82. Available at: http://dx.doi.org/10.1016/j.techfore.2014.07.014.

Bundesamt für Statistik BFS, 2017. Struktur der Schweizer KMU 2015. Statistik der Schweiz.

Chan Kim, W. \& Mauborgne, R., 2005. Blue Ocean Strategy: How to Create Uncontested Market Space and Make Competition Irrelevant 1st ed., Harvard Business Review Press.

Ewaldsson, U., 2016. Five trends: Shaping innovation in ICT. Ericsson Review, 93(2), pp. 30-41.

Gatzweiler, A., 2013. Technologie Portfolio Analyse., p. 15.

Hevner, A.R. et al., 2004. Design Science in Information Systems Research., 28(1), pp. 75-105.

Kimber, A. et al., 2015. Emerging technology trends - The road to the bank of the future. Emerging technology trends The road to the bank of the future, p. 8.

Kreutzer, R.T., Neugebauer, T. \& Pattloch, A., 2018. Digital Business Leadership, Berlin, Heidelberg: Springer Berlin Heidelberg. Available at: http://link.springer.com/10.1007/978-3-662-56548-3.

Marques, M., 2017. 5 tech trends revolutionising the hospitality industry. Medium. Available at: https://medium.com/hijiffy/5-tech-trends-revolutionising-the-hospitality-industry-76b734c961b7 [Accessed September 30, 2018].

Paine, J., 2018. Don't Miss these 5 Retail Tech Trends in 2018. Inc. Available at: https://www.inc.com/james-paine/ top-retail-tech-trends-to-watch-in-2018.html [Accessed September 30, 2018].

Panetta, K., 2017. Top Trends in the Gartner Hype Cycle for Emerging Technologies. Gartner Inc. Available at: https://www.gartner.com/smarterwithgartner/top-trends-in-the-gartner-hype-cycle-for-emerging-technologies-2017/ [Accessed September 30, 2018].

Parsons, R. et al., 2018. Technology Radar Vol. 19. thoughtworks.com/radar, 19. Available at: https://www.thoughtworks.com/radar [Accessed October 30, 2018].

Pelz, W., 2004. Strategisches und Operatives Marketing: Ein Leitfaden zur Erstellung eines professionellen Marketing-Plans 1st ed., Norderstedt. Available at: http://www.wpelz.de/swot-analyse/SWOT-Analyse.pdf.

Peter, M. et al., 2018. Die ABILI-Methodik : Inspiration und Navigation bei der Digitalen Transformation mit Fokus auf KMU. Arbeitsberichte der Hochschule für Wirtschaft FHNW, (36).

Peter, M.K., 2017. KMU-Transformation: Als KMU die Digitale Transformation erfolgreich umsetzen. 1st ed., Olten: Marc K. Peter.

Rohrbeck, R., Heuer, J. \& Arnold, H., 2006. The Technology Radar - An instrument of technology intelligence and innovation strategy. ICMIT 2006 Proceedings - 2006 IEEE International Conference on Management of Innovation and Technology, 2(June), pp. 978-983.

Theobald, E., 2016. PESTEL-Analyse: Die Wichtigsten Einflussfaktoren der Makroumwelt. Management Monitor, pp. 1-7. 